\section{ACADEMIC HEALTH CENTERS: WILL THEY SURVIVE?}

Dramatic changes in the provision of US health care loom large on the horizon. Maintaining the missions of education, research, and clinical service for Academic Health Centers (AHCs) remains essential as new care paradigms unfold. Health reform measures designed to reduce health care spending will likely place further stress on AHCs. ${ }^{1}$ Are AHCs capable of responding? Current evidence suggests that the future of AHCs and their ability to fulfill their joint missions are at risk.

Research, particularly research supported by the National Institutes of Health (NIH), is the sine qua non of any respected $\mathrm{AHC}$, but external research funding does not generally cover all costs. Additional funds are required through margins from clinical services and philanthropy.

AHCs provide a significant amount of medical education on the medical student, graduate medical education, and continuing education levels. As with research, medical education requires additional funding that must be generated from clinical care margins, donations, and state support. As clinicians confront increasing economic pressures, their availability to participate in medical education is increasingly limited. ${ }^{2}$ AHCs may be hard pressed to come up with the resources needed, particularly as calls are made to reform medical education to make it more learner- and patient-centered. ${ }^{3}$

At a time when financial demands on medical schools and their AHC partners are escalating, their funding may be in jeopardy. Medical schools and AHCs are subject to the uncoordinated priorities of the NIH, donors, and state and federal governments.

The current situation is more dire because all of these metrics are moving in the wrong direction simultaneously, at least when considered from the viewpoint of the AHC. ${ }^{4}$

How should AHCs respond? One potential option for AHCs is to embrace practice transformation: Accountable Care Organizations (ACOs) and the Patient Centered Medical Home (PCMH). While there is real evidence that the Mayo Clinic, Geisinger Health System, Kaiser Permanente, the Cleveland Clinic, and others are making the transition to an $\mathrm{ACO}$, there are few examples of AHCs. ${ }^{5}$ The same can be said for the medical home. While both ACOs and medical homes are heavily dependent on primary care, most AHCs have not committed resources to this underfunded but necessary part of the health care system. ${ }^{6}$ In addition, as a group they have not increased the number of trainees in primary care specialties.

In the 1990s, the AAMC's Generalist Initiative, in concert with the Robert Wood Johnson Foundation ${ }^{9}$ was a significant effort with the intent to stimulate interest in the primary care disciplines and coincided with the first phase of managed care. Real increases in the number of primary care residency positions and a surge in popularity of primary care specialties followed., ${ }^{7,8}$

Over the past 18 months, several efforts have been made to engage with the current leadership of the AAMC and others on the essential nature of primary care and the maintenance of key missions of AHCs. Despite these efforts, we remain deeply concerned that AHCs appear to be failing to recognize the importance of primary care physicians as essential to the success of their educational and research missions. Both missions are placed in jeopardy as larger health care organizations are rapidly evolving through the development of ACOs based on primary care providers. Those organizations will compete much more effectively on price and accessibility, will add specialty providers that will successfully complete with AHCs, leaving them as niche providers and with seriously weakened research and educational capabilities. This would be a serious loss from which we would have difficulty recovering. Concrete action is needed.

Russell Robertson, MD, and the Association of Departments of Family Medicine

This commentary was reviewed and edited by the ADFM Executive Committee.

\section{References}

1. Bach PB. Quality wrapped in volume inside a hospital. Ann Intern Med. 2009;150(10):729-730.

2. Barsansky B, Kenagy G. The full-time clinical faculty: What goes around comes around. Acad Med. 2010;85(2):260-265.

3. Lindor KD, et al. Commentary: improving medical education during financially challenging times. Acad Med. 2010;85(8):1266-1268.

4. Tallia AF, Amenta PS, Jones SK. Commentary: academic health centers as accountable care organizations. Acad Med. 2010;85(5):766-767.

5. Joiner KA, Libecap A, Cress $A E$, et al. Supporting the academic mission in an era of constrained resources: approaches at the University of Arizona College of Medicine. Acad Med. 2008;83(9):837-844.

6. Robert Wood Johnson Foundation. The Generalist Physician Initiative. 2001. http://www.rwjf.org/pr/product.jsp?id=17971.

7. Pugno $P$, et al. Results of the 2008 national resident matching program. Fam Med. 2008;40(8):563-573.

8. Altarum Institute. Modeling the specialty mix of new physicians: policy implications for influencing the primary care supply. June 2010. 